Revue d'histoire de l'Amérique française

REVUE D.HISTOIRE DE L'AMÉRIQUE FRANÇAISE

\title{
Le syndic de la colonie acadienne en Poitou
}

\section{Pierre Massé}

Volume 5, numéro 1, juin 1951

URI : https://id.erudit.org/iderudit/801684ar

DOI : https://doi.org/10.7202/801684ar

Aller au sommaire du numéro

Éditeur(s)

Institut d'histoire de l'Amérique française

ISSN

0035-2357 (imprimé)

1492-1383 (numérique)

Découvrir la revue

Citer cet article

Massé, P. (1951). Le syndic de la colonie acadienne en Poitou. Revue d'histoire de l'Amérique française, 5(1), 45-68. https://doi.org/10.7202/801684ar d'utilisation que vous pouvez consulter en ligne.

https://apropos.erudit.org/fr/usagers/politique-dutilisation/ 


\section{LE SYNDIC DE LA COLONIE ACADIENNE EN POITOU}

Expérience unique en son genre que celle-ci. Non que l'Histoire ait ignoré, avant le XVIIIe siècle, les transferts de population dont une époque récente ne nous a donné que trop d'exemples. Mais l'installation des Acadiens en Poitou se situe à un moment crucial. La Révolution est toute proche, avec ses conséquences sociales qui vont remettre en question tout le passé de ce coin de France, faire disparaître le promoteur de la colonie, apporter une nouvelle échelle des valeurs. Avec, aussi, ses conséquences économiques dont les répercussions s'étendront jusqu'au fond des brandes. La communauté acadienne, introduite dans l'organisme poitevin en 1773, va-t-elle se comporter comme ces corps étrangers qui s'enveloppent d'une mince pellicule isolante pour mener leur vie à part? Ou laissera-t-elle la substance environnante pénétrer peu à peu, par osmose, la sienne propre? Autrement dit, comment se sont comportés les Acadiens après avoir accepté, une fois pour toutes, de se fixer sur notre sol?

Le premier trait de leur activité qui doive nous retenir est l'ensemble de leurs opérations agraires. Tout d'abord les défrichements. La colonie comprend, selon le mot d'un agronome contemporain, "des sols qui se classent certainement parmi les plus mauvais de notre pays"1. Essarter cette terre ingrate, lui apporter les éléments qui lui manquent, en faire une région dont, aujourd'hui, la fertilité surprend: les Acadiens réussissent, au XVIIIe siècle, une véritable création humaine en substituant un nouveau sol à leur sol infécond. A l'encontre de tout déterminisme géographique, l'homme a imposé ici sa volonté à la terre ${ }^{2}$. Il nous importe de savoir comment il y est parvenu.

1. Ernest Baillargé, Les milieux et les productions agricoles dans la Vienne (Poitiers, 1932), 31. - Voir également Régions géographiques de la France (Institut national de la Statistique et des étrides ́́conomiques. Ministère de l'Education nationale, Paris, 1950), 305.

2. Le Lannou, La gégraphie humaine (Paris, 1949). 
De savoir aussi le temps qu'a demandé cette création. De préciser la cadence des défrichements. Parmi les 57 métairies installées dans les brandes poitevines, lesquelles ont progressé le plus vite, et pourquoi? Au départ, l'immense tapis de bruyères est le même pour tous. Chaque exploitant part de zéro. Lequel prend la tête, et qui reste le dernier?

C'est dire que l'aspect social du problème double l'aspect agraire et parfois le conditionne. L'inévitable sélection des intelligences, des initiatives, puis des alliances et des fortunes, a clivé de bonne heure le groupe acadien. Quels autres facteurs ont accéléré la différenciation des familles? Et, de ces familles, lesquelles sont demeurées fidèles à leur nouvelle petite patrie, tandis que d'autres ne songeaient qu'à s'en évader?

A toutes ces questions, comme à celles qui se lèveront, parfois de façon inattendue, lorsque nous pénétrerons dans la vie de la communauté, nous essaierons d'apporter des réponses basées sur des textes précis. Le plus souvent, ces derniers seront des minutes de notaires $^{3}$. Car les Acadiens ont beaucoup trafiqué durant les années qui suivirent leur installation. Si le sol de leur colonie fut une création agraire, la répartition de ce sol, et son amodiation, furent une création économique, au sens complet du mot. Alors que les baux à ferme et à colonnage de la région sont figés, depuis des siècles, dans des clauses identiques, certains des baux acadiens apportent quelques formules nouvelles, parfois déconcertantes par leur caractère insolite. L'exégèse que nous en proposerons repose sur de nombreuses analogies avec des cas tout semblables, issus des mêmes conditions sociales qui se retrouvent aussi bien dans le passé médiéval qu'au siècle dernier.

Or, les annales de la colonie acadienne, à partir du moment où cette colonie s'organise en vue de l'avenir, font souvent état d'un individu qui domine son temps et son milieu: le syndic. La chronique de ce personnage se confond souvent de façon étroite avec l'histoire du groupe. Souvent àussi, elle la dépasse. Choisi par ses pairs pour défendre leurs intérêts, le syndic ne négligera pas pour autant les

3. Nous avons l'agréable devoir de remercier ici Me Parthenay, notaire et Conseiller général de Vouneuil-sur-Vienne, et Me Riguet, notaire à Archigny (Vienne) qui nous ont réservé le meilleur accueil en nous permettant de dépouiller leurs archives. Chez Me Parthenay se trouvent les minutes Drouault, Gauvain, Vézier et Amirault; chez Me Riguet, les minutes Bridnne et Collet. 
siens propres. $N$ 'aurait-il point tendance, en tant que chef de clan, a exploiter un peu ses protégés, tout en luttant pour eux? Parti, lui aussi, de la table rase, n'a-t-il pas reçu, pour le démarrage de ses defrichements, quelque forte impulsion qui lui a permis de prendre et de garder une sérieuse avance? Cette vitesse acquise, l'a-t-il transmise en héritage à ses enfants ? Ceux-ci l'ont-ils, à leur tour, conservée?

Mais il faut d'abord nous demander qui était cet homme. Et c'est là qu'une première surprise nous attend. Le syndic des Acadiens n'était pas un Acadien.

LE SYNDIC DES ACADIENS:

SON ROLE; SON MARIAGE

AVEC MARIE-REINE BERBUDEAU; SA FAMILLE

Avant d'aller plus loin, il nous paraît indispensable de résumer en quelques mots les circonstances qui ont amené des familles acadiennes à s'installer dans les brandes incultes du Haut-Poitou, sur les communes d'Archigny, Monthoiron La Puye et Saint-Pierre de Maille.

Chassés d'Acadie par les Anglais sous Louis XV, des milliers de colons furent débarqués en France. Le Marquis de Pérusse d'Escars, qui vivait alors à Monthoiron où il avait tenté d'importants défrichements, recueillit, en 1773, d'accord avec le gouvernement français, des exilés acadiens sur ses terres. On bâtit, pour recevoir les arrivants, 57 maisons dont un certain nombre s'aligne de part et d'autre d'une voie nouvelle, percée au milieu des brandes, et qui porte encore ce nom caractéristique: La Ligne. A chaque maison devait être attribué un mas de terre vierge de 176 boisselées, suffisant pour la faire vivre.

Les projets de Pérusse d'Escars ne purent soutenir l'épreuve des faits. Dès 1775 , trois groupes d'Acadiens abandonnaient successivement la colonie et partaient en direction de Nantes, quelques-uns pour la Louisiane. Nouveaux départs en 1776, sans compter quelques exodes individuels. Au total, sur les 1472 Acadiens attirés en Poitou, il n'en resta que 157 , pour lesquels 57 maisons étaient disponibles. 
On n'avait pas à craindre la crise du logement. A telles enseignes que certaines de ces habitations furent données à des non-Acadiens, notamment à deux anciens militaires: les sieurs Brionne et Guillard de la Vacherie, et que la demoiselle Pérusse d'Escars, pour sa part, en reçut 19 par un arrêt du Conseil d'État du 26 juillet 1785.

Pendant longtemps, le seul travail d'ensemble sur les Acadiens du Poitou fut celui du général Papuchon, datant de 1908, fort honorable pour l'époque, mais d'une documentation succinte ${ }^{4}$. Il appartenait à M. Ernest Martin, en 1936, de nous donner le livre complet qui, dans une langue souple et ferme, traite magistralement l'aspect historique de la question acadienne ${ }^{5}$. L'ensemble des pièces justificatives qu'il publie en appendice ou dans le corps du volume constitue la charte des Acadiens. Nous allons y trouver le statut du syndic.

Dès 1775, Turgot envisageait la nomination, par l'Intendant du Poitou, de trois syndics, dont chacun régenterait 18 ou 20 maisons et serait choisi par une assemblée d'habitants. Le projet ne put prendre corps, et l'Intendant se borne à charger, en 1777, Brionne, l'ancien soldat que nous avons rencontré tout à l'heure, "de veiller au bon ordre de la colonie". Et c'est seulement en 1783 que des lettres-patentes fixent le régime de la communauté. L'article 13 stipule, en effet, que, tous les trois ans, un syndic sera élu par le groupe pour y exercer la juridiction, maintenir la police, et suivre les affaires conformément aux lois.

De ces élections, n'est demeurée aucune trace. Nous savons seulement que le même syndic resta en fonctions jusqu'à la Révolution qui le supprima ipso facto par la création des Municipalités, le 14 décembre 1789. Mais cette fonction est tellement acquise et reconnue que, longtemps après qu'elle a pris fin, son détenteur en conserve le titre aux yeux des autorités. En 1792, il est appelé, par le notaire, "syndic des Acadiens" ; en l'an IV et en l'an VI, par le Juge de Paix, "fondé de pouvoirs des Acadiens"; en l'an IX, par le Sous-Préfet,

4. Général Papuchon, La colonie acadienne du Poitou. (Bulletin de la Société des Antiquaires de l'Ouest, Poitiers, 1908), 2e trimestre.

5. Ernest Martin, Les exiles acadiens en France au XVIIIe siècle, et leur établissement en Poitou (Paris, 1932).

6. Minutes Amirault.

7. Archives de la Vienne, L 526 à 535. 
"syndic de la colonie acadienne"s. Témoignages d'une considération qui persiste à travers les régimes et nous conduit inévitablement à soulever la question suivante: pourquoi ce personnage fut-il choisi ailleurs que chez les colons?

Pérusse d'Escars, qui avait, comme tous les gens du XVIIIe siècle, le sens aigu des nuances sociales et avait soin de mettre chacun à son rang, n'applique la dénomination de sieur, strictement réservée aux bourgeois, qu'à un seul Acadien. Agissent de même le notaire et le curé, experts en la matière. C'est ainsi que nous est révélée la préséance dont jouit le sieur Jean Gabriel Berbudeau, "ancien chirurgien major de la marine et subdélégué de l'Isle Saint-Jean et isles adjacentes"'. Il est aussi "pensionné de Sa Majesté"10 et touche, à ce titre, 300 livres de rente, somme importante. On l'a doté d'un cheval pour visiter les malades de la colonie ${ }^{11}$. L'homme-médecine, là comme ailleurs, est installé aux premières places.

Le sieur Berbudeau vit avec sa femme, Marie Gervais Paris et leur fille Marie-Reine, au hameau Saint-Antoine, paroisse d'Archigny. Avait-il recueilli chez lui sa nièce Magdeleine Renault, dont les parents étaient morts? L'orpheline épousa, le 2 mars 1778, un Acadien, René Mélançon, veuf de Marie-Louise Gibon ${ }^{12}$. Le ménage disparaît bientôt, attiré par Nantes et la Louisiane. On constate également la présence d'un Alexandre Berbudeau, dont nous ne savons s'il a quelque parenté avec le médecin. Il est de ceux qui, en 1775, demandent à s'en aller. Pérusse d'Escars le range parmi "les familles qui, sous aucun prétexte ne doivent être maintenues en Poitou ni leur départ retardé"13. Formule qui en dit long.

Par contre, la considération que Pérusse d'Escars témoigne au chirurgien transparaît clairement dans une lettre écrite à Hérault, subdélégué de l'Intendant à Châtellerault, le 4 août 1775. Dictée à

8. Ibid., Carton 28, No 5.

9. Archives commmunales d'Archigny. Registres paroissiaux.

10. Archives de la Vienne. E 4, 16 bis 62 .

11. Ernest Martin, Les exilés canadiens en France au XVIIIe siècle, et leur établissement en Poitou (Paris, 1932), 777.

12. Registres paroissiaux d'Archigny.

13. Archives de la Vienne, J 64. 
un secrétaire, cette lettre contient, en post-scriptum, quelques lignes, aisément reconnaissables comme étant de la main même du marquis. Spécialement consacrées à Berbudeau, elles montrent l'importance que tient le médecin dans les préoccupations de Pérusse ${ }^{14}$. De telle sorte que, joignant le prestige du bourgeois et du guérisseur à l'aisance du pensionné de l'État, et possédant l'estime du seigneur de Monthoiron qui était aussi un des grands seigneurs de France, tout désignait Berbudeau pour représenter l'es Acadiens. Mais, en 1783, lorsqu'on dota la colonie d'un syndic, il avait 73 ans. Et l'ambition, peut-on croire, ne l'animait pas. Quelqu'un de son entourage immédiat, par contre, n'en était pas dépourvu.

Deux ans auparavant, en 1781, sa fille, Marie-Reine Berbudeau, avait trouvé un mari. Elle n'était pas la première Acadienne à épouser un garçon du pays. Cette particularité avait été réservée à Marie Boudrot, orpheline, "fille majeure de feu Pierre Boudrot et de feue Cécile Véco tous les deux jadis habitant l'Acadie et actuellement nouvellement établis dans cette paroisse"15. D'autres épousailles avaient suivi, où le marié était inévitablement un laboureur ou un journalier. Mais, pour la famille Berbudeau, il ne pouvait être question d'aller manger le gâteau des noces dans une chaumine enfumée. La fille d'un bourgeois ne pouvait épouser qu'un fils de bourgeois.

Celưi qui se présenta remplissait la condition essentielle. Avec, en plus, quelque chose d'extrêmement rare et précieux dans ce coin de France. Pierre Alexis Texier de la Touche était licencié ès lois. Nous ne connaissons, dans les environs, qu'un personnage du même grade: de Lauzon, sénéchal de Chauvigny. Nous ne sommes pas sûrs que Hérault, subdélégué de l'Intendant, ait possédé ce titre, et tout nous porte à croire que son fils, Jacques François Hérault, qui fut Receveur des Domaines sous la Révolution et l'Empire, ne l'avait pas non plus ${ }^{16}$.

Le père de Pierre Alexis était, lui aussi, un sieur: Bernard Texier de la Touche, demeurant au bourg de Bonneuil-Matours avec sa

14. Ibid.

15. Registres paroissiaux d'Archigny.

16. Archives de l'Enregistrement et des domaines, Poitiers. Nous avons plaisir a remercier ici $M$. Lefranc, directeur de ce service, qui a bien voulu chercher pour nous le renseignement. 
femme, Françoise Le Beau. Lorsque fut décidé le mariage avec l'Acadienne, ce fut Emery Gauvain, notaire royal à Vouneuil-surVienne, qui dressa le contrat, le 20 octobre 1781. Pierre Alexis de la Touche apportait en dot une borderie sise en Boirie, hameau de Bonneuil-Matours, avec une maison d'habitation au même endroit, plus une rente annuelle et perpétuelle de 4 livres due à Bernard Texier par un certain Boumard. Le tout estimé 640 livres.

Les Berbubeau donnaient à leur fille une des maisons qu'ils possédaient au hameau Saint-Antoine où le jeune ménage résiderait désormais. Le mariage se faisait sous le régime de la communauté, chaque conjoint apportant 100 livres pour commencer ${ }^{17}$. Et, le mois suivant, le 27 novembre 1781, les noces étaient célébrées dans l'église d'Archign ${ }^{18}$.

Elles furent malheureusement, à peu de distance, suivies d'un deuil. Le 15 décembre 1781, Marie-Reine enterrait sa mère âgée de 60 ans $^{19}$ Femme morte et fille mariée, le médecin Berbudeau restait seul. Nous n'entendrons plus parler de lui. Le dernier acte qu'il nous ait laissé, peu avant son décès, est une procuration en blanc pour toucher sa pension ${ }^{20}$. Le prestige du vieil homme ira à son gendre, le licencié ès lois redevenu paysan, qui, installé au cœur de la colonie, aimant la vie rurale, poussera vigoureusement son exploitation. Il est si bien naturalisé Acadien qu'en 1783 il est élu syndic et le restera sans désemparer.

Le jour de son mariage, il a 29 ans et sa femme 22 . Il est né à Bonneuil-Matours, le 2 septembre 1752. Elle, nous ignorons où elle vit le jour. Quelle sera la destinée de ce jeune couple qu'une singulière conjoncture a réuni? Il est bien difficile de ne pas épiloguer sur cette rencontre, dans les brandes d'Archigny, d'un ancien élève de l'École de Droit et d'une Française d'au delà des mers. Comment se sontils connus? Quand bien même une telle question, et la précédente, seraient de celles qui ne doivent pas longtemps retenir, on ne peut les empêcher de s'ébaucher à l'arrière-plan de l'esprit. Autant vaut, dans

17. Archives de la Vienne. E 4, 16 bis, 62.

18. Registres paroissiaux d'Archigny.

19. Ibid.

20. Minutes Amirault. 
ce cas, les aborder de front. Essayons donc de déchiffrer les filigranes, qui, à travers le papier timbré des actes notariés, nous apportent parfois des éléments d'une étonnante précision sur le psychisme des individus. C'est en étudiant de près les origines de Pierre Alexis Texier, et plus particulièrement le milieu familial, que nous y parviendrons.

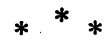

L'accession de Bernard Texier de la Touche au titre honorifique de sieur était, quand son fils se maria, de date relativement récente. La famille était originaire d'Archigny où elle habitait le minuscule hameau de la Touche, dont la toponymie est révélatrice d'anciennes sylves. Une touche en Haut-Poitou désigne un ensemble de grands et beaux arbres. Non loin de cet habitat, on remarque les maisons appelées la Jarrie, de garric (chêne), la Forêt, la Brousse, appellations qui parlent d'elles-mêmes. On trouve des Texier à la Touche pendant tout le XVIIIe siècle et sous l'Empire. Établir leur parenté avec les Texier de Bonneuil-Matours ne serait qu'une affaire de recherches dans les registres paroissiaux des deux communes voisines. Nous ne l'entreprendrons point. Des probabilités comme celles que nous allons trouver, chemin faisant, semblent suffisantes.

Le voisin le plus rapproché des Texier, à Archigny, était, à la fin du XVIIIe siècle, "le sieur Anselme Beaudet des Roches, bourgoiss, demeurant en sa terre de Joline". Il possède la Brousse, qu'il a louée, le 20 mars 1787, à Louis Boumard, journalier, et la Forêt, louée, le 4 décembre de la même année, à Pierre Alexis Epin ${ }^{21}$. Beaudet des Roches a épousé la fille de Chardon, chirurgien d'Archigny et cousin des Texier. Son fils, Pierre Alexis, baptisé le 26 mars 1784, aura pour parrain Pierre Alexis Texier ${ }^{22}$. Nous avions été amenés a nous demander tout à l'heure comment le futur syndic des Acadiens, né à Bonneuil-Matours et y vivant, avait pu connaître sa femme. Un simple coup d'œil sur la carte nous montre la proximité de la Touche, berceau des Texier, avec la fameuse Ligne, chemin des Acadiens. Les Texier de Bonneuil avaient conservé, sinon des biens, du moins des

21. Minutes Brionne.

22. Registres paroissiaux d'Archigny. 
liens de famille à la Touche. Des intérêts aussi, puisque la rente de 4 livres mise dans la corbeille de noces par Bernard Texier était due par Louis Boumard, métayer de la Brousse. Le bourgeois Beaudet des Roches est lui-même redevable d'une rente plus importante, se montant à 27 livres, envers le bourgeois Bernard Texier. Voilà donc éclairé en partie un premier poịt: le garçon qu'épousa Marie-Reine Berbudeau appartenait à une vieille dynastie du voìsinage, où des motif́s d'ordre familial ou pécuniaire étaient de nature à le ramener.

Deuxième question, assez facile à résoudre, en raison de l'abondance et de la précision des documents. Quelle était la situation de fortune de Bernard Texier, et quel rang occupait ce bourgeois dans l'échelle sociale au moment où il maria son fils?

Dès le 27 février 1749, un indice probant nous est donné. On baptise, ce jour-là, dans l'église de Bonneuil-Matours, Louis Bernard, fils de Bernard Texier, qui n'est pas encore sieur, et de Françoise Le Beau. Le parrain et la marraine sont, l'un et l'autre, d'une très vieille famille noble, venue d'Aunis à la fin de la Guerre de Cent ans: messire Louis de Marans, seigneur de Saint-Mars, et demoiselle de Marans. Signe évident de la place que le père du nouveau-né tient déjà dans la paroisse. L'enfant meurt le 14 septembre de l'année suivante. Pierre Alexis, qui naît ensuite, aura pour parrain, le 2 septembre 1752, Pierre Guignard, futur maire de Bonneuil-Matours, et sa femme Marie-Anne Sénéchaux ${ }^{24}$. Sur le berceau des enfants Texier ne se sont pas penchés des petites gens.

C'est que la profession de Bernard confère à son possesseur, dans le village et ses alentours, une autorité exceptionnelle. BonneuilMatours est situé à l'orée de la forêt de Moulière, qui étend son imposant massif sur les terrains de transport tertiaires argilo-sableux où elle couvre 7947 arpent $\mathrm{s}^{25}$. Bernard Texier en est l'un des gardes. A lui d'y interdire jalousement l'usage des pratiques millénaires que la Maîtrise des Eaux et Forêts s'efforce de combattre: le braconnage, l'érussage des feuilles convoitées pour la nourriture des bestiaux, les coupes clandestines de bois, le ramassage des glands - ou de fermer les yeux s'il le juge à propos.

24. Archives communales de Bonneuil-Matours. Registres paroissiaux.

25. Archives Nationales. N II Vienne 1. 
Ses gages, certes, sont assez minces. En 1780, il n'a touché que 90 livres pour toute l'année, auxquelles il faut ajouter 26 livres 15 sols de droit de chauffage et 16 livres pour l'assistance aux veuves ${ }^{26}$. Car il est aussi, dans une certaine mesure, dispensateur de secours. Mais il a une autre source de revenus. Tandis qu'il court les bois, Françoise Le Beau tient un commerce. Bernard Texier est désigné comme marchand ${ }^{27}$. Nous apprendrons plus tard où était situee sa boutique et ce que l'on pouvait y trouver.

Lui, a d'autres préoccupations que de vendre de la chandelle. Nous l'avons vu créancier d'une rente de 4 livres due par Boumard, d'une de 27 livres due par Beaudet des Roches. Il en possède bien d'autres, acquises ou constituées entre 1771 et l'an III, et que lui payent des personnages les plus divers: Jacques Forêt, marchand; Girard, sabotier; Mathieu Roy, chirurgien; André Delaveau, sacristain; Charles Charaudeau, laboureur. Il touche une autre rente des Carré de Busseroles, ancienne famille noble de Vouneuil-sur-Vienne, de François La Gauderie, important bourgeois de Chênevelles qui sera plus tard député suppléant à la Convention ${ }^{28}$. Il veille soigneusement à ce que les notaires en dressent des titres nouvels, c'est-à-dire une reconnaissance consentie par le débiteur lorsqu'il y a décès ou mutation.

Avec l'argent vient la terre. Et surtout cette terre organisée, agencée, faite pour le rapport immédiat, qui s'appelle la métairie. Toutefois, cette métairie, dont l'achat est le signe irréfutable de la richesse, ne sera pas, et ne pourra jamais être assise dans les sols féconds de la plaine alluviale. La noblesse rurale, depuis plus d'un siècle, s'est emparée de toutes les fermes étalées sur les limons quaternaires de la Vienne, dans la varenne. Bernard Texier devra se rabattre sur les exploitations plus récentes du plateau, gagnées à coups de defrichements: les borderies. Il en possède trois, de part et d'autre de cette vallée interdite aux bourgeois, sur les limites du finage. A l'est, la Grève, qui contient encore, en 1810, une importante proportion de brandes: 4 hectares pour 20 hectares de labours ${ }^{29}$. A

26. Archives Nationales. $R^{1} 256$.

27. Minutes Vézier.

28. Minutes Drousult, Gaurain, Vézier.

29. Archives communales de Bonneuil-Matours. Cadastre de 1810. 
l'opposé, vers l'ouest, en Boirie, les bâtiments et terres qu'il donna en dot à son fils. Tout près enfin, de la forêt de Moulière, le Charbon blanc, dont il faut réchauffer chaque année, à grandis renforts de marne, le sol froid.

Scindées en trois tronçons éloignés l'un de l'autre, les borderies du sergent-garde ne sont pas de première qualité. Mais les autres bourgeois de la paroisse ont été servis de même façon. Claude Soin de la Savinière, qui s'est donné seulement la peine de naître pour devenir sieur, et ne fait rien de ses dix doigts que d'acheter, vendre, arrenter et affermer, gère des exploitations du même ordre. L'oasis de la Vienne, et ses fertiles terres à blés, n'accueille que les fortunes nobiliaires. La métairie de la Guillonnière, au cœur de la varenne, acquise par Pérusse d'Escars en 1756, fút payée 8,600 livres. La métairie de Mauvais Vent, qui équivaut à la Grève et lui est symétrique dans l'espace, est vendue 800 lívres à la même époque ${ }^{30}$. Voilà qui délimite la position tenue par Bernard Texier dans la paroisse. Notre homme, sorti d'un modeste hameau d'Archigny, s'est hissé, par son travail, son commerce et son esprit d'entreprise, dans la classe bourgeoise de Bonneuil-Matours. Au moment où les Acadiens arrivent, il est déjà un sieur, et fait suivre son nom du nom de son pays d'origine. A la Révolution, il supprimera cette particule qui n'est plus de mise et s'appellera Texier-Latouche ${ }^{31}$. Entre temps, la famille continue l'ascension avec le licencié ès lois, et avec la sœur de celui-ci, une maîtresse femme dont nous n'avons pas encore parlé.

L'acte de baptême de Marie-Anne Texier de la Touche nous est inconnu. Pierre Alexis, le futur syndic des Acadiens, n'est âgé que de onze ans lorsque sa sœur épouse, le 8 février 1763, Jean Autexier, originaire de Fressineau où son père est marchand ${ }^{32}$. Le jeune marié s'installe à Bonneuil-Matours et, à l'instar de son beau-père, y ouvre une boutique. Rapidement, il se lance dans les transactions de la terre et devient ce que l'on appelle au XVIIIe siècle un fermier, c'est-à-dire un homme d'affaires. Ces métairies de la plaine alluviale auxquelles les hommes de sa classe sociale ne peuvent accéder, il

30. Archives de la Vienne. Dépôt 22, liasse 219.

31. Y. Godecho't, "L'aristocratie des titres bourgeois", (Annales historiques de la Revolution française, No 120, (octołbre-décembre 1950), 368.

32. Registres paroissiaux de Bonneuil-Matours. 
arrive quand même à les avoir sous sa coupe. Il les obtient en location de la marquise d'Hargicourt, leur propriétaire, par groupes de trois. Après quoi, il les sous-loue, une à une, par un bail à mi-fruits, à quelque laboureur ${ }^{33}$. Disons, en langage moderne, que Jean Autexier est un intermédiaire, et ajoutons qu'il n'y perd point.

Le ménage est prolifique. Trois enfants naissent coup sur coup, en 1764, 1765, 1766; d'autres s'espacent en 1769, 1771, 1774, 1776, 1780, 1785. Deux filles meurent et deux se marient ${ }^{34}$. Cependant, le père est devenu un personnage important de la paroisse. Collecteur des tailles en 1773, le voici syndic de Bonneuil-Matours, quelque chose comme le maire de l'époque, et on l'appelle sieur à son tour ${ }^{35}$. Distinction honorifique bien précaire. Il est à peine âgé de 50 ans qu'on le porte en terre, le 25 octobre $1789^{36}$. Marie-Anne Texier reste avec la boutique, les métairies de madame d'Hargicourt et cinq enfants sur les bras.

Accumuler tous ces détails ne nous écarte point des Acadiens, dont les descendants mêleront leur sang à celui des descendants du sergent forestier et du marchand de Fressineau. Le comportement de Marie-Anne Texier, après la mort de son mari, est aussi à retenir, en ce qu'il nous permet de comprendre celui de son frère, le licencié ès lois.

La veuve se met à la besogne. Elle s'adapte vite aux choses de la terre, conserve les métairies, en surveille l'exploitation. Le 2 pluviose an III, elle afferme encore de madame d'Hargicourt les trois métairies des Roches, "suivant le dernier bail fait à son mari" et venu à expiration, pour trois ans, moyennant 3000 livres "en argent monnaie ayant cours" ${ }^{37}$. Avec la dépréciation des assignats, son loyer ne lui reviendra pas trop cher.

Les notaires et l'officier d'Etat-Civil lui donnent maintenant le titre de fermière. Pour ses litiges, elle a choisi un procureur de premier ordre en la personne d'Habert, administrateur du District, qui sou-

33. Archives de la Vienne, dépôt 22, liasse 184.

34. Registres paroissiaux de Bonneuil-Matours.

35. Minutes Drouault.

36. Registres paroissiaux de Bonneuil-Matours.

37. Archives de la Vienne, dépôt 22, liasse 185. 
tiendra ses intérêts devant le Tribunal de Commerce ${ }^{38}$. Ses achats de terres se poursuivront pendant tout l'Empire. C'est seulement à la Restauration, où elle donne procuration à son fils, en 1819 qu'elle pense à se reposer ${ }^{39}$.

Telle était la famille où entra Marie-Reine Berbudeau. Le vieux chirurgien de l'Ile Saint-Jean, demeuré sagement dans ses brandes lors de l'exode de ses compatriotes pour Nantes, ne pouvait avoir d'inquiétude quant à l'avenir de sa fille. Il avait atteint 82 ans lorsqu'il mourut, le 4 janvier 1792, à Archigny, "dans son habitation de la colonie acadienne formée dans cette paroisse" ${ }^{40}$. On l'enterra le lendemain. Quelques-uns de ses camarades du Grand Dérangement, entre autres Marin Daigle, l'accompagnèrent au petit cimetière de village où nous n'avons pu, un siècle et demi plus tard, malgré toutes nos recherches, le retrouver.

\section{II}

\section{LE GROS GEUVRE DU SYNDIC: BORNAGE DE LA COLONIE ACADIENNE}

Ainsi la disproportion est frappante entre la famille Texier de la Touche, qui accède à la bourgeoisie avec des terres au soleil, des maisons, des relations étendues au monde rural de deux paroisses, et la famille Berbudeau, riche seulement de son passé et de son avenir. Le présent se résumant pour elle en deux bâtisses qu'entourent, à perte de vue, des brandes où il faudra créer de toutes pièces une nouvelle exploitation.

Ce n'est donc pas un mariage de raison, prévu et organisé selon l'usage de l'époque, que celui du 27 novembre 1781. Deux ans plus tard, quand seront parues les lettres patentes fixant le statut de la colonie, une jeune fille acadienne pourra devenir un bon parti. L'article 5 de ces ordonnances stipule, en effet, que "les familles acadiennes établies ou à établir, et ceux qui s'uniront à elles par mariage ou

38. Ibid., liasse 450.

39. Minutes Amirault.

40. Registres paroissiaux d'Archigny. 
autrement, seront exemptes pendant l'espace de trente années de toutes tailles, impositions, capitation, vingtièmes et autres impositions territoriales". Ajoutons-y l'exemption des dîmes ecclésiastiques pendant cinq ans à partir du ler janvier 1780 (article 2). Le facteur intérêt a pu jouer un rôle dans les unions qui suivirent ces lettres patentes. Dans celles qui les précédèrent, s'il intervint jamais, ce fut seulement à l'arrière-plan.

Six enfants naquirent au jeune ménage, entre 1782 et 1791 , à Archigny. Deux moururent, l'un en 1783 , l'autre en $1786^{41}$. Il resta quatre filles. Nous en connaîtrons plus tard la destinée.

Celle de Pierre Alexis Texier de la Touche, pour l'instant, tient dans son horizon de brandes. Si large qu'en soit la perspective, à travers le plateau monotone de Monthoiron, encore est-il bon de connaître avec exactitude la place dévolue à ses voisins. Et c'est là que le licencié ès lois aura l'occasion de mettre en pratique ses connaissances juridiques, attendu que personne ne peut se vanter d'indiquer sans se tromper ses propres limites. Dans l'installation de la colonie, on n'a oublié qu'une chose: le bornage des propriétés.

Avant que Texier fût marié et installé parmi eux, quelques-uns des Acadiens se sont élevés contre cet état de fait. Personne n'étant là pour les représenter et les défendre, ils prennent eux-mêmes leurs intérêts en mains et poussent jusqu'à Poitiers où ils vont protester auprès de l'Intendant. "Ambroise Guillot et Marin Daigle sont venus me voir il y a quelques jours, écrit Blossac à Pérusse d'Escars en avril 1780, pour me dire que des propriétaires de la paroisse d'Archigny inquiétaient les colons de trois habitations de la colonie voisine de cette paroisse, prétendant qu'on y avait englobé des terrains qui leur appartenaient, même qu'une de ces habitations avait été construite sur leur terrain; ils m'ont ajouté que vous avez eu connaissance de ces prétentions et que vous aviez fait espérer aux réclamants qu'on leur donnerait d'autres terrains en échange. J'ai promis à ces Acadiens de vous en écrire. Ce serait un objet à terminer le plus tôt possible" ${ }^{\prime 2}$. Il est inutile d'ajouter que ces affaires d'empiètement deviendront une magnifique source de procès.

41. Registres paroissiaux d'Archigny.

42. Archives de la Vienne, dépôt 22, liasse 124. 
C'est le même Marin Daigle qui se plaint, le lundi 28 mars 1791, des envahissements de son voisin Pierre Merle, un laboureur de Cenant. Le Juge de Paix, devant qui est portée l'affaire, décide que Merle ne devra plus, "à l'avenir, bécher un chaume ou pacage qui appartient au dit Daigle comme faisant partie de la maison et dépendances qu'il possède en la colonie acadienne, paroisse d'Archigny, et qui fut au nommé Harbour, aussi Acadien" "43. Précisons que ce Harbour, qui avait pour femme Marie Horé, ne resta point dans la colonie et partit vraisemblablement pour Nantes. Le 2 juin 1778, sa femme avait mis au monde un fils, baptisé le lendemain et parrainé par le chirurgien Josnon. On est surpris, quelques années après le départ du père, de trouver trace de l'enfant, mort en $1782^{44}$. Que ces exodes successifs aient disloqué bien des familles acadiennes, ne doit point nous étonner. En l'an XII, on parlait encore de "la maison qu'occupait Harbour" ${ }^{\widetilde{4}}$. Pendant son séjour à Archigny, cet Acadien n'avait pas dû passer inaperçu.

Procès, aussi, pour la possession des habitations. Les départs, suivis bientôt de retours repentants, créent des situations singulières. François Daigle et Jeanne Hoby, vieux ménage acadien, abandonnent les brandes inhospitalières pour la Louisiane, entraînant avec eux leur fille Marie-Thérèse et son mari René Baudeau, un jeune homme d'Archigny épousé dans l'intervalle. Le No 29 qu'ils habitaient, devenu vacant, est attribué à la demoiselle Pérusse d'Escars. Jusqu'au jour où Baudeau, souffrant sans doute du mal du pays, revient de Nantes avec son Acadienne et bat sa coulpe pour se faire pardonner l'équipée. Ce retour des enfants prodigues attendrit le seigneur de Monthoiron, qui restitue la maison confisque $e^{46}$. Mis en goût par cette libéralité, Baudeau, quelques années plus tard, réclame une autre "maison et dépendances acadiennes qui fut à François Daigle, son beau-père, absent depuis 7 ans" et où est installé Ambroise Guillot que nous connaissons déjà.

A l'audience du 11 avril 1791, le Juge de Paix constate "que la concession authentique de cette maison, si aucunes il y a, n'a pas été

43. Ibid., liasse 564 .

44. Registres paroissiaux d'Archigny.

45. Minutes Collet.

46. Archives de la Vienne, dépôt 22, liasse 124. 
présentée par les parties"47. L'attendu du magistrat est très net. Les titres de possession, en Acadie poitevine, avaient les mêmes lacunes que la propriété terrienne. Le maquis des brandes se doublait d'un maquis de la procédure, non moins difficile à pénétrer.

Comme réplique à l'action en justice que Baudeau vient de lui intenter, Ambroise Guillot attaque à son tour un autre Baudeau et deux autochtones appelés Tranchand et Texier, qui comparaissent, le 6 août 1792, accusés d'usurpation. Le juge ordonne une visite des lieux pour le samedi 11 aout suivant. Sur le terrain, on est bien obligé de reconnaître que l'espace contesté ne dépend pas de la colonie. On a retrouvé, en effet, le titre primitif, vieux d'un'siècle et demi, portant bail de 176 boisselees, mesure de Chauvigny', consenti le 17 juin 1648 à un maréchal nommé Fulgent Chicard, et confirmé par titre nouvel le 30 janvier 1767. Aucun doute, par conséquent, quant à la possession des terres que l'on peut aisément localiser. Ambroise Guillot se voit débouté de sa demande et condamné aux dépens, soit 5 livres, y compris 2 livres pour le transport de justice ${ }^{48}$.

Les connaissances juridiques de Texier de la Touche le mettrontelles à l'abri des mêmes erreurs? C'est ce que nous allons voir.

Les No 17 et 18 attribués à Berbudeau étaient construits en partie sur un terrain appartenant à Gabriel Sauvion, laboureur d'Archigny, partie sur un autre appartenant à Sylvain Robin, laboureur à Bonnes et Alexis Vachon, de Chauvigny. Pour quelles raisons Sauvion, qui résidait dans la paroisse et avait dû assister à cet empiètement, n'a-t-il pas réagi? Pérusse d'Escars ou l'Intendant lui ont-ils laissé entrevoir des compensations? Ou faut-il voir l'explication de son silence dans le fait que les intérêts de sa famille se confondaient maintenant avec ceux des Acadiens?

Le 27 novembre 1780, en effet, notre laboureur mariait sa fille Marie à Paul Guillot, fils de cet Ambroise Guillot que nous connaissons déjà. En même temps, par un de ces mariages doubles que l'on rencontre assez souvent à l'époque, il mariait son fils Jean à Anne-Gertrude Guillot, sœur de Paul. Le double contrat, fort modeste, avait été dressé le 14 précédent ${ }^{49}$. Ajoutons que Françoise,

47. Ibid., liasse 564 .

48. Ibid.

49. Registres paroissiaux d'Archigny; Archives de la Vienne. E 4, 6-36 (17). 
une autre fille de Sauvion, avait épousé, à une date inconnue, François Merle, laboureur de la région. Retenons la composition de ce brelan d'héritiers, où figurent un frère et une sœur acadiens, car nous allons souvent le retrouver.

Sauvion mort, ses trois descendants se départirent de la tolérance manifestée jusqu'alors par le père à l'égard de Texier-Latouche, et ouvrirent les hostilités. Ils réclamèrent le remboursement de la pièce de terre prise par les No 17 et 18, et le conflit eut son dénouement devant le notaire, le 28 février 1791.

Ces terres consistaient en 60 bqisselées de brandes dont l'origine est indiquée sans équivoque. "Ledit défunt Sauvion les avait abandonnées en faveur de l'établissement qu'il a plu au Roy de faire en 1773 pour différentes familles acadiennes, et sur laquelle pièce il fut construit alors 2 habitations qui ont été accordées au sieur Berbudeau et à la défunte son épouse, Acadiens'. Des 60 boisselées, Berbudeau et son gendre avaient défriché 40 . Pour être admis à les conserver, Texier-Latouche devra verser 600 livres aux co-héritiers.

L'affaire ne va pas sans discussion, puisqu'il avait été question - nous l'avons vu par la lettre de Blossac - de dédommager les propriétaires ayant donné des terres à la colonie. Les possesseurs auraient perçu, sur ces pièces, un droit de champart. Clause que l'on ne retrouve nulle part dans les dispositions officielles, et dont l'absence prouve, une fois de plus, l'incertitude où tout le monde était plongé. "On s'était mis à l'œuvre, écrit M. Ernest Martin, sans prendre la peine de rédiger un cahier des charges véritables. Il y avait bien eu des conversations, des mémoires échangés entre le marquis de Pérusse et les ministres, mais sans aucun contrat formel liant toutes les parties"50. Le projet de champart est à ranger, sans doute, avec les nombreuses maquettes crayonnées à l'âge héroïque de l'installation acadienne.

Quoi qu'il en soit, Texier-Latouche prend ses précautions et fait spécifier que ses $\mathbf{4 0}$ boisselées seront aff ranchies de tout champart, rente ou prestation. Il reconnait seulement qu'elles sont mouvantes de la seigneurie de Monthoiron, et sujettes au devoir de 4 boisseaux d'avoine et 4 deniers, et encore arrive-t-il, par on ne sait quel marchandage, à faire contribuer ses vendeurs, pour l'avenir, au verse-

50. Ernest Martin, op. cit., 197. 
ment de la moitié environ de ces droits ${ }^{51}$. Les uns et les autres n'eurent pas à les payer longtemps.

De toute la pièce de brandes possédée par feu Sauvion, il restait encore 20 boisselées disponibles. Jean Sauvion et Anne-Gertrude Guillot vendirent, le 27 décembre suivant, leur part, soit le tiers, à Texier-Latouche, moyennant 66 livres. Pour ses deux achats de cette même année 1791, le syndic devait faire borner et limiter les parcelles $^{52}$. Formalité dont l'importance apparaîtra bientôt.

Ayant indemnisé les héritiers Sauvion de leurs 40 boisselées de terre sur lesquelles étaient bâtis les No 17 et 18, Texier pouvait se croire désormais à l'abri de toute revendication. S'il eut jamais cette assurance, il dut déchanter le 24 nivôse an II, jour où le trio revint à la charge, bien décidé à lui soutirer encore quelque argent.

Remettant en question l'acte du 28 février 1791, ils prétendirent que les terres avaient été vendues très au-dessous de leur valeur, et qu'ils étaient lésés de la moitié du prix réel. "Ils sont fondés, suivant la loy, à rentrer en possession d'iceux objets en remboursant la dite somme de 600 livres avec tous les frais et loyaux coûts". Y eutil pression d'ordre politique sur le bourgeois, à une époque où les Comités révolutionnaires de village pouvaient se permettre, nouvellement constitués, des interventions dans bien des domaines? C'est le $30 \mathrm{du}$ même mois que se forme le Comité de Vouneuil-sur-Vienne dont l'activité, de tous les groupements semblables de la région, est la mieux connue ${ }^{53}$. Que fut celui d'Archigny? Nous en ignorons tout. Constatons seulement qu'il n'y eut pas procès. Texier cède sur toute la ligne, ce qui n'est guère dans ses habitudes. Il verse les 600 livres supplémentaires réclamées par les trois héritiers ${ }^{54}$, qui lui confèrent enfin le droit de se croire chez lui.

Ces difficultés ne l'empêchèrent pas de renouer, les années suivantes, des transactions avec une famille qui lui avait donné tablature, mais dont il avait encore besoin. Des brandes de feu Sauvion, les deux-tiers, soit environ 13 boisselées, restaient invendues et con-

51. Minutes Brionne.

52. Ibid.

53. Archives de la Vienne, liasse 314.

54. Ibid., E 4, 16 bis, 64. 
tinuaient à intéresser Texier-Latouche comme avoisinant ses propres terres. Il lui fallut, néanmoins, attendre quelque temps avant de les annexer. Le 19 ventôse an V, Paul Guillot et Anne Sauvion lui vendirent leur part, c'est-à-dire 6 boisselées $1 / 2$ payées comptant $t^{55}$. Enfin, un an a près, jour pour jour, il achetait le dernier tiers, de François Merle et Françoise Sauvion, pour 78 livres $^{56}$. La main du syndic, après beaucoup de mal, se refermait enfin, en dernier ressort, sur le bien du vieux laboureur.

De même qu'il avait transigé avec les héritiers Sauvion, Texier dut négocier en face de Sylvain Robin de Bonnes, et Alexis Vachon, de Chauvigny. Ils lui reprochèrent, les 14 mars et 16 mai 1791, d'avoír "empiété sur une grande pièce de terre appartenant aux dits Robin et consorts appelée la Prise à Grateau", traversée par le chemin de Châtellerault à Saint-Savin. Des réclamants, Vachon était le plus précis. "Le sieur Texier, dit-il, ou quoi que ce soit le sieur Berbudeau, son beau-père", ont pris 6 boisselées en deux morceaux, et doivent les payer. A quoi le syndic répond que les objets réclamés par Vachon sont enclavés dans les terres de la colonie acadienne, et demande l'exhibition des titres de propriétés. La conclusion de cette affaire nous apprend que, là aussi, Texier dut s'incliner ${ }^{57}$. "Pour ne pas perdre le fruit de ses travaux, il a transigé à gros frais avec les uns et avec les autres"58.

Pareille mésaventure advint au sieur Guillard de la Vacherie, ancien officier au Normandie Infanterie, détenteur de deux maisons acadiennes, les Nos 39 et 40 , accordées pour l'indemniser des pertes qu'il avait éprouvées au service du roi. En lui faisant ce cadeau, le 22 septembre 1783, le Conseil d'État avait seulement omis de s'assurer si les terres si généreusement offertes ne dépendaient point de quelqu'un d'autre. Elles relevaient, effectivement, d'un seigneur poitevin, M. de Chasteigner, à cause de sa terre de Marsujault, à Archigny, dont le No 39 faisait partie. Les droits féodaux se montaient, chaque année, à 15 boisseaux de seigle et trois quarts de

55. Minutes Amirault.

56. Ibid.

57. Archives de la Vienne, liasse 564.

58. Ibid., dépôt 22, liasse 124. 
boisseaux d'avoine, mesure d'Angles, 7 poulets, 4 chapons et 36 sols 9 deniers de cens. Avec des arrérages accumulés, bien entendu.

Le nouveau propriétaire eut un jour la désagréable surprise de s'entendre réclamer, non seulement les devoirs nobles en retard, mais encore une déclaration ou titre nouvel portant reconnaissance de ces droits pour l'avenir. Force lui fut donc de composer avec le seigneur ${ }^{59}$. On peut croire qu'il apprit sans regret l'abolition des droits féodaux. Nous allons le retrouver dans un instant, et nous verrons que cet ancien officier s'était parfaitement adapté aux questions acadiennes qui avaient si mal commencé pour lui.

Pour sortir de ces situations invraisemblables et de celles qui ne pouvaient manquer de surgir, une seule solution: borner les terrains. Ce fut le gros œuvre de Texier-Latouche, qui avait de bonnes raisons pour s'y employer.

Entre temps, son autorité avait dépassé le cadre acadien, et s'étendait maintenant à la paroisse tout entière. Dans la première municipalité d'Archigny, élue au début de 1790, il était procureur de la commune. Détail significatif: en dressant la liste des nouveaux promus, et en relatant leurs délibérations, le greffier lui applique l'épithète de sieur et ne l'applique à aucun autre, pas même au maire François Cloué. C'est lui, Texier, qui, le 19 juillet 1790, convoque l'assemblée au grand complet avec la garde nationale où, dans ce pays de brandes, on est un peu surpris de trouver un colonel, un commandant, un capitaine, un adjudant et un sergent-major. C'est lui qui dresse les rôles d'impôts de 1791, alors qu'un peu partout le District de Châtellerault est obligé d'envoyer des émissaires pour faire ce travail que les nouvelles municipalités sont bien incapables d'exécuter. Le syndic des Acadiens se comporte un peu, au début de la Révolution, comme le syndic d'Archigny. Pour devenir officiellement le chef et le représentant de la commune, il ne lui reste plus qu'à être élu maire. C'est chose faite en $1793^{60}$.

Fort de sa position politique, soutenu par toute la colonie, en même temps qu'aiguillonné par son intérêt personnel, Texier-Latouche s'adressa au Directoire du département de la Vienne pour demander le bornage, ou, comme on disait alors, le bornoiement des

59. Ibid.

60. Archives de la Vienne, liasse 406. 
terrains. Ce qui fut accordé le 23 mai $1792^{61}$. Avec ou sans discussion préalable? Les procès-verbaux des séances du Directoire sont malheureusement absents des archives départementales pour le premier semestre de 1792 , lacune qui nous prive d'attendus dont on eât aimé connaître le détail.

L'arpenteur, Vincent Amirault, n'avait jamais eu si belle occasion d'exercer son ministère. Habitant Monthoiron, où il vivait dans le sillage fructueux de Pérusse d'Escars, il apparaît, lui aussi, comme un sieur, et non des moindres. C'est un des très rares personnages de cette histoire sur lequel on possède quelques détails physiques: "cheveux blonds châtain, yeux bleus, front découvert". Comme beaucoup de ses contemporains, il est grêlé. "Visage marqué de la petite vérole," dit son passeport ${ }^{62}$. Cette disgrâce imméritée ne l'empêche pas d'épouser, en 1789, la demoiselle Marie Ribreau. Mariage de bourgeois, où l'épouse apporte 609 livres, l'époux, 1000 livres $^{63}$. On rencontre Amirault dans les nombreux arpentements de fréresches que Pérusse d'Escars et La Roche du Maine font effectuer, de 1785 à 1789 , pour se faire payer des arrérages que les prestataires oublient volontiers ${ }^{64}$. Il est peu de brandes sur lesquelles il n'ait promené sa toise et son calepin.

Celles-ci vont l'occuper longtemps. Pendant plus d'un an, Amirault mesurera, visera, taillant à pleins ciseaux dans le drap neuf de la colonie. Avec des arrangements, des accommodements entre les parties intéressées, car il est des endroits défrichés que les laboureurs entendent bien garder pour eux. D'aucuns s'opposent même à l'arpentage, et le syndic des Acadiens aura bien du mal à mettre tout le monde d'accord.

C'est ici que réapparaît le sieur Guillard de la Vacherie. Les difficultés qu'il a connues avec le No 39 n'existent plus depuis que. sont abolies les prestations féodales, mais d'autres l'attendent avec le No 40 appelé aussi Petit Dépôt. La malchance s'acharne sur cet ancien officier. Établi à la Guadeloupe avec sa femme, il fut capturé

61. Ibid., E 4, 16 bis, 50.

62. Ibid., liasse 407.

63. Minutes Brionne,

64. Minutes Drouault, Vézier. 
par les Anglais tandis qu'il retournait en France, emmené en captivité à Guernesey, et laissa toute sa fortune dans l'aventure. Les deux propriétés qu'on lui donna en compensation, voici qu'il lui faut maintenant les défendre l'une après l'autre, puisqu'en fait elles sont loin de lui appartenir intégralement.

Deux de ses voisins, en effet, vont jusqu'à dire que les terres du No 40 ne sont pas à lui, mais à eux-mêmes. Il s'agit de Louis de la Bussière, ex-seigneur du Chillou, où il habite, et de son beau-frère, Louis Vézien de Champagne, qui réside à Bauroy, près de Saint-Savin. Sûrs de leurs droits, ils ont fait cultiver les terres du Petit Dépôt. Mieux encore, ils les ont encloses et fermées de fossés, entendant marquer par là qu'ils sont bien chez eux.

Procès. Guillard fait citer La Bussière et Champagne devant le Juge de Paix de Pleumartin. Les accusés répondent que les terres en question avaient été arrentées à leurs parents par l'Évêché de Poitiers, et que l'arrentement est toujours valable. En attendant que soit dénoué ce nœud gordien, intervient, le 28 février 1792, une sentence provisoire, maintenant Guillard dans la jouissance de ses deux maisons et dépendances, et ordonnant aux deux voisins de combler les fossés dans le courant du mois.

Jusqu'alors, la discussion ne dépasse pas l'horizon du No 40. Mais voici qu'elle risque d'entraver sérieusement les opérations de Vincent Amirault. Car La Bussière et Champagne forment opposition au bornoiement et n'entendent pas que l'arpenteur vienne opérer sur des terres dont ils persistent à revendiquer la possession.

Comment sortir de cette impasse? Devenu plus que jamais nécessaire, le bornage ne peut être mené à bien si des intéressés lui font de l'obstruction. L'ex-syndic, dénommé maintenant "fondé de pouvoirs de la colonie acadienne", va s'efforcer de mener à bien une conciliation difficile. Situation paradoxale: le No 40 a deux propriétaires, l'un et l'autre de bonne foi et tous les deux décidés à ne pas céder. On peut faire confiance, heureusement, au licencié ès lois, maintenant rompu aux fonctions qu'il exerce depuis neuf ans, pour trouver une solution.

Cette dernière a lieu devant notaire, au No 18, domicile de Texier-Latouche, le 27 novembre 1792. Elle est laborieuse, et il semble bien que Guillard de la Vacherie, une fois encore, ait fait les frais de l'opération. 
Il faut croire que les titres d'arrentement, consentis jadis par l'Évêché de Poitiers, sont en bonne et due forme, et point entachés de féodalité, puisque leurs détenteurs s'engagent à les remettre à Guillard, qui sera ainsi en pleine possession du Petit Dépôt. Il recevra le terrain nécessaire pour compléter les 176 boisselées qui doivent revenir a chaque habitant de la colonie. Mais pas gratuitement.

La Bussière et Champagne n'abandonnent leurs biens qu'à deux conditions. La première est que les deux tiers des terres concédées seront essartées et cultivées dans le cours des 12 années qui vont suivre, le tiers restant pouvant être gardé en brandes ou pacages. Cette clause est comminatoire. A défaut de son exécution, les deux beaux-frères reprendront possession des maisons et terrains sans que l'on puisse s'y opposer.

Pourquoi s'intéressent-ils de la sorte à la mise en culture de territoires dont ils n'auront plus désormais à s'occuper? Simplement et c'est la deuxième condition - parce qu'ils entendent bien continuer à en tirer profit, et pour longtemps. La terre de Guillard sera grevée d'un terrage au $1 / 16$ des fruits ${ }^{65}$. Autrement dit, l'ex-officier sera toute sa vie tributaire de ses voisins, détail que le Conseil d'État de 1783 n'avait sûrement pas prévu. La propriété foncière, en ce qui concerne le No 40, n'est qu'un fermage déguisé. Nous verrons plus tard ce qu'il en advint et qui, de La Bussière et Champagne ou de Guillard, eut le dernier mot.

L'essentiel, pour le syndic des Acadiens, était que cette affaire se terminât, et que le bornage pât enfin être effectué. Commencé le 12 juin 1792, il était clos le 8 juin 1793. De bonne heure, ce jour-là, à 6 heures du matin, le No 18 vit à nouveau une réunion de personnages qui se penchèrent sur un procès-verbal. La rédaction dura toute la matinée, de 6 à 11 heures. Texier-Latouche signa "tant pour moy et mon épouse que pour les parties intéressées au procès-verbal, absentes". Les 10,736 boisselées de terres étaient partagées ${ }^{66}$.

On aimerait savoir qui paya les frais de cette longue, vaste et délicate entreprise. Probablement les propriétaires, comme nous le donne à croire une quittance qu'Amirault délivra à Laideck, le 9

65. Archives de la Vienne. E 4, 16 bis, 50.

66. Minutes Drousult. 
frimaire an II "pour sa part et portion des frais de mesurage, bornage et plan de la colonie acadienne, à cause de l'habitation No 31 a luy appartenant"67. Mais l'établissement de la facture et les détails du remboursement nous échappent encore.

Les ciseaux d'Amirault, dans leur découpage rigide des brandes, en ont-ils fixé l'assemblage une fois pour toutes? Nous allons le savoir en étudiant les premières années de la colonie, avec la mise en valeur de ses premiers établissements. Ce qui nous doit retenir maintenánt, c'est, au sein de cette colonie, l'activi té personnelle du syndic. Grâce à Texier-Latouche, s'est réglée la grosse affaire collective. Que seront ses affaires particulières, notamment dans ses rapports avec les hommes qui ont mis en lui leur confiance et l'ont choisi pour représentant?

$$
\text { (à suivre) }
$$

Pierre Massé, Paris, France

67. Archives de la Vienne, liasse 530. 